\begin{tabular}{lllll} 
CVC- & 380 & 300 & 40 & 40 \\
Colon & & & & \\
CVC- & 612 & 492 & 60 & 60 \\
Clinic & & & & \\
\hline
\end{tabular}

Conclusions WCE plays an essential role in diagnosis and prevention of colorectal cancer. However, distinguishing abnormal WCE images with polyps is a significant challenge because it needs high time cost. The results show that our method can help physicians analyse the WCE images and reduce their pressure. This method could be further utilised in the clinical trials to help physicians from the tedious image analysing work.

\section{IDDF2018-ABS-0258 A CASE REPORT OF WARFARIN INDUCED SPONTANEOUS SUB-MUCOSAL AND MESENTERIC HEMATOMA IN THE GASTROINTESTINAL TRACT (SMALL BOWEL) CAUSING BOWEL OBSTRUCTION}

Manuel Jr Velasco*, Mark Anthony De Lusong, Yvonne Mina. Victor R Potenciano Medical Center, Philippines

\subsection{6/gutjnl-2018-IDDFabstracts. 179}

Background To present a rare case of Warfarin-induced spontaneous sub-mucosal and Mesenteric hematoma in the Gastrointestinal Tract (Small Bowel) causing bowel obstruction.

Methods Case Report.

Literature Review.

Results A case of a 67-year-old male is presented with a chief complaint of abdominal pain for three days and difficulty passing stool. He has a known case of Hypertensive atherosclerotic cardiovascular disease, Rheumatic Heart disease, Coronary Artery Disease s/p Coronary Angiography, Chronic Atrial Fibrillation, and Diabetes Mellitus Type II. Maintained on several medications including ASA $80 \mathrm{mg} 1 \mathrm{tab}$ OD and
Coumadin $5 \mathrm{mg} 11 / 2 \mathrm{tab}$ OD. He was lost to follow-up for one year. On his admission, his PT and INR were reportedly undetectable. He was admitted and treated as a case of Small Bowel Ischemia secondary to spontaneous sub-mucosal and Mesenteric haemorrhage and Non- ST Elevation Myocardial Infarction. The entire duration of the hospitalisation took 11 days to include ICU stay, and surgical procedure and recovery. Conclusions This case highlights the risk of haemorrhage in a high-risk patient taking warfarin and the need for close monitoring and follow-up. An intramural hematoma should be entertained in patients on warfarin treatment presenting with clinical signs and symptoms of bowel obstruction. Non-operative treatment of spontaneous small bowel hematoma has a good outcome in most patients. This also stresses the importance of diligent follow-up with the attending physician to ensure a proper balance of anticoagulation and risk of bleeding. On the other hand, this case emphasises that over-anticoagulation leading to bleeding creates negative feedback to the heart, by increasing metabolic demands, which can lead to a paradoxical thromboembolic phenomenon such as Myocardial

\section{IDDF2018-ABS-0259 SEGMENTATION OF INTESTINAL POLYPS VIA A DEEP LEARNING ALGORITHM}

${ }^{1}$ Liansheng Wang*, ${ }^{1}$ Yuqi Qian, ${ }^{2}$ Yanxing Hu. ${ }^{1}$ Xiamen University, China; ${ }^{2}$ Xiamen Innovision Medical Technology Company, China

\subsection{6/gutjnl-2018-IDDFabstracts. 180}

Background Colorectal is the third most commonly occurring cancer in men and the second most commonly occurring cancer in women. At least $80 \%-95 \%$ of colorectal cancers are evolved from intestinal polyps. Therefore early screening of intestinal polyps is one of the crucial ways to prevent colorectal cancer. WCE is widely used in the examination of the internal environment of the digestive tract. However, lots of medical images per patient undoubtedly create a considerable burden on doctors. Thus, in this paper, we describe a
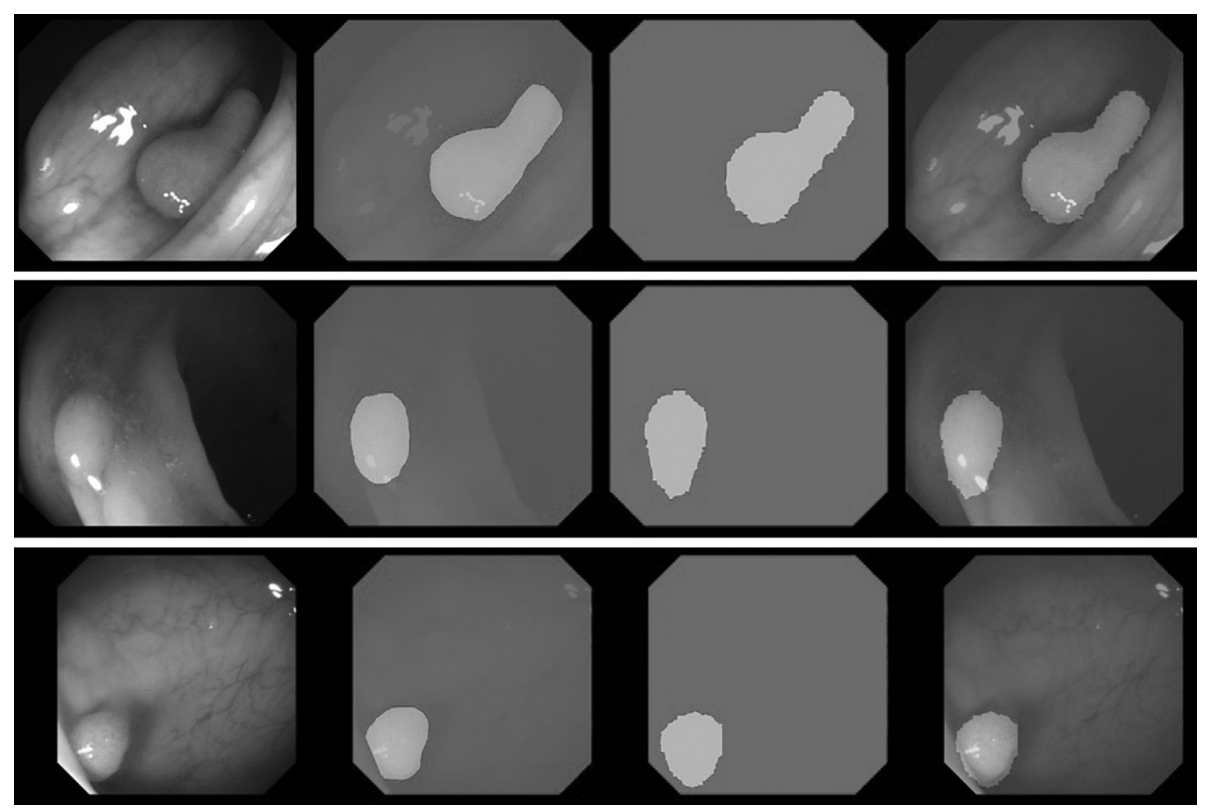

Abstract IDDF2018-ABS-0259 Figure 1 Examples of segmentation results on the test data. Each column represents a single frame, a ground truth annotation, a prediction image, and a prediction image annotation 